\title{
An approximate method for solving complex Ginzburg-Landau equation
}

\author{
Xiang Chunhuan ${ }^{1, a}$, Wang Honglei ${ }^{2, b^{*}}$
}

${ }^{1}$ School of Public Health and Management, Chongqing Medical University, Chongqing, P.R. China

${ }^{2}$ College of medical informatics, Chongqing Medical University, Chongqing 400016, P.R. China

aw8259300@126.com, bw8259300@163.com, "Corresponding author

Keywords: First integral method, Complex Ginzburg-Landau equation, Evolution solutions, Traveling wave solutions.

Abstract. The first integral method is applied to solve complex Ginzburg-Landau equation in this work. The evolution solutions for the equation are obtained. This method is based on the theory of commutative algebra, which can be applied to nonintegrable equations as well as to integrable ones. The first integral method supplied an effcient way to obtain traveling wave solutions of some nonlinear partial differential equations. This approach can also be applied to other nonlinear fractional differential equations.

\section{Introduction}

Nonlinear partial differential equations are widely used to describe complex phenomena in various fields of science. The generalized complex Ginzburg-Landau equation is one of the most-studied nonlinear equations in the physics community, which arises from a wide variety of fields, such as quantum field theory, superconductivity, superfluidity, Bose-Einstein condensation, liquid crystals and strings in field theory[1, 2, 3]. In optics, this equation (or a generalization of it) describes the essential features of processes in lasers $[4,5,6,7,8]$. Some of the numerical techniques designed under the non-standard methodologies have been popularized in the literature: tanh-sech method[9, 10, 11], Jacobi elliptic function expansion method[12], hyperbolic function method[13], Riccati equation method[14], F-expansion method[15]. The problem of finding first integrals of ordinary differential equations (ODEs) was initially considered by Darboux and by Lie.[16]

The first integral method is based on the ring theory of commutative algebra[17], which is widely used by many such as in $[18,19]$ and by the reference therein and this approach can also be applied to other nonlinear fractional differential equations. We applied the first integral method for the complex Ginzburg-Landau equation and the evolution solutions

\section{The first-integral method}

For a given nonlinear evolution equation with two variables in the form

$$
P\left(u, u_{t}, u_{x}, u_{t t}, u_{x x}, u_{x t}, u_{x x x}, \ldots\right)=0 \text {, }
$$

where $u=u(x ; t), P$ is a polynomial $u$ and its derivatives. Using a wave variable $\xi=x+\lambda t$, Eq.(1) is changed into ordinary differential equation, which can be rewritten as

$$
Q\left(u, u^{\prime}, u^{\prime \prime}, u^{\prime \prime \prime}, \ldots\right)=0 \text {. }
$$

The prime in Eq.(2) denotes the derivative with respect to the same variable $\xi$. The equation $u(x, t)$ $=f(\xi)$ is supposed to be the solutions of Eq.(2). Then, a new independent variable is read as the following Eq.(3), which leads a system of nonlinear ordinary differential equations Eq.(4). With the

$$
\begin{aligned}
& X(\xi)=f(\xi) ; \quad Y=\frac{\partial f(\xi)}{\partial \xi}, \\
& Y=\frac{\partial X(\xi)}{\partial \xi} ; \quad \frac{\partial Y(\xi)}{\partial \xi}=F(X(\xi), Y(\xi))
\end{aligned}
$$

same conditions, the general solutions to Eq.(4) can be obtained directly by employing the qualitative theory of ordinary differential equations [17]. However, in general, it is really difficult for us to realize 
this even for one first integral, because for a given plane autonomous system, there is no systematic theory that can tell us how to find its first integrals, nor is there a logical way for us to tell what these first integrals are. Fortunately, the first integral for Eq.(4) is obtained by employed the Division Theorem, which can help us obtain one first integral for Eq.(4) which reduces Eq. (2) to a firstorder integrable ordinary differential equation. The evolution solution for Eq.(1) is finally obtained by solving the first-order integrable ordinary differential equation.

The Division theorem for two variables in the complex domain $\mathbf{C}$ is read as: Division theorem: Suppose that $G 1[w ; z], G[w ; z]$ are polynomials in $C[w ; z]$ and $G 1[w ; z]$ is irreducible in $C[w ; z]$. If $G[w ; z]$ vanishes at all zero points of $G 1[w ; z]$, then there exists a polynomial $G 2[w ; z]$ in $C[w ; z]$ such that

$$
G[w ; z]=G 1[w ; z] G 2[w ; z]
$$

\section{Complex Ginzburg-Landau Equation}

The The complex Ginzburg-Landau equation, which is one of the most-studied nonlinear equations in the physics community, is studied in this section. The complex Ginzburg-Landau equation is read as:

$$
u_{t}=u-(1+i b)|u|^{2} u+(1+i a) u_{x x}
$$

where $a$ and $b$ are constant parameters, $u$ is the function of $x$ and $t$. By using the transformation $u(x ; t)$ $=e^{\theta} f(\xi), \xi=x+\lambda t$ and $\theta=\alpha x+\beta t$, where $\lambda, \alpha$, and $\beta$ are constants, the ordinary differential equation transferred from Eq.(6) is obtained

$$
f_{\xi \xi}+\frac{2 i \alpha-2 \alpha a-\lambda}{1+i a} f_{\xi}+\frac{1-\alpha^{2}-i a \alpha^{2}-i \beta}{1+i a} f-\frac{1+i b}{1+i a} f^{3}=0 .
$$

With the help of Eq.(3), we obtain

$$
\dot{X}=Y ; \quad \dot{Y}=\frac{1+i b}{1+i a} X^{3}-\frac{2 i \alpha-2 \alpha a-\lambda}{1+i a} Y-\frac{1-\alpha^{2}-i a \alpha^{2}-i \beta}{1+i a} X .
$$

According to the first-integral method and Division theorem, we suppose that $X(\xi)$ and $Y(\xi)$ are the nontrivial solutions of Eq. (8). $G(X ; Y)$ can be expressed as ${ }^{G(X, Y)}=\sum_{i=0}^{m} a_{i}(X) Y^{i}=0$. The above expression is an irreducible polynomial in the complex domain $C[X ; Y]$, then the above equation with parameter $\xi$ is rewritten as

$$
G(X(\xi), Y(\xi))=\sum_{i=0}^{m} a_{i}(X(\xi)) Y^{i}(\xi)=0
$$

where $a_{i}(X(\xi)),(i=0 ; 1 ; 2 ;::: m)$ are polynomials of $X$ and $a_{m}(X(\xi)) \neq 0$. Eq.(9) is called the first integral for Eq. (8). According to the Division Theorem and the derivative of $G$, a polynomial $g(X)+h(X) Y$ in the complex domain $C[X ; Y]$ must meet the relationship

$$
\frac{d G}{d \xi}=\frac{d G}{d X} \frac{d X}{d \xi}+\frac{d G}{d Y} \frac{d Y}{d \xi}=(g(X)+h(X) Y) \sum_{i=0}^{m} a_{i}(X) Y^{i}
$$

Here, two different cases, $m=1$ and $m=2$ are assumed in Eq.(9) in this example.

Case A. Expanding Eq.(10) and equating the coefficients of $Y i,(i=0 ; 1 ; 2)$ on both side of with the parameter $m=1$, we have Eq. $(11,12,13)$

$$
\begin{aligned}
& a_{1}(X)=h(X) a_{1}(X) \\
& a_{0}(X)=g(X) a_{1}(X)+h(X) a_{0}(X)-a_{1} \frac{2 i \alpha-2 \alpha a-\lambda}{1+i a} \\
& g(X) a_{0}(X)=a_{1}(X)\left(\frac{1+i b}{1+i a} X^{3}-\frac{1-\alpha^{2}-i a \alpha^{2}-i \beta}{1+i a} X\right) ;
\end{aligned}
$$

From Eq.(9), we known that $a_{i}(X)(i=0,1)$ are polynomials, then $a_{1}(X)$ is constant and $h(X)=0$ are deduced from Eq.(11). For simplicity, $a_{1}(X)$ take the form $a_{1}(X)=1$. Balancing the degrees of $g(X)$ and $a_{0}(X)$, we conclude that $\operatorname{deg}(g(X))=1$ only. Suppose that $g(X)=A_{0}+A_{1} X$ with parameter $A_{0}$ and $A_{1}$ to be determined, then we find $a_{0}(X)$ read as 


$$
a_{0}(X)=\frac{1}{2} A_{1} X^{2}+A_{0} X-\frac{2 i \alpha-2 \alpha a-\lambda}{1+i a}+R,
$$

where $R$ is some an integration constant. Substituting $a_{1}, g$ and $a_{0}$ into Eq.(13) and setting all the coefficients of powers $X$ to be zero, then we obtain a system of algebraic equations and by solving it, we obtain

$$
A_{1}=\sqrt{\frac{2(1+i b)}{1+i a}} ; \quad A_{0}=\frac{2(2 i \alpha-2 \alpha a-\lambda)}{3(1+i a)} ; \quad R=0
$$

where $\lambda, b, \alpha, a$ and $a \neq i$ are constants. Using the conditions Eq.(15) and Eq.(9), we obtain the evolution solution of the Eq.(6)

$$
\begin{gathered}
\lambda=\frac{2-2 i \beta-2 \alpha^{2}-2 \alpha^{2} a^{2}}{a \alpha} ; M_{1}=\frac{2 i(x+\lambda t) \alpha}{3(1+i a)}+4 a \alpha \sqrt{1+i a}+2 \lambda \sqrt{1+i a} M_{2}=\frac{2 a(x+\lambda t) \alpha}{3(1+i a)}+4 i \alpha \sqrt{1+i a}+\frac{(x+\lambda t) \lambda}{3(1+i a)} \\
f(x, t)=-\frac{2 e^{M_{1}} \sqrt{1+i a}(2 i \alpha-2 a \alpha-\lambda)}{e^{M_{2}}-3 \sqrt{2(1+i b)} e^{M_{1}}(1+i a)} \quad u(x, t)=e^{\alpha x+\beta t} f(x, t) .
\end{gathered}
$$

Case B. Equating the coefficients of $Y i,(i=0 ; 1 ; 2 ; 3)$ on both side of Eq.(10) with $m=2$, we obtain

$$
\begin{aligned}
& a_{2} \dot{(X)}=h(X) a_{2}(X) \\
& a_{1}(X)=h(X) a_{1}(X)+g(X) a_{2}(X)-2 a_{2} \frac{2 i \alpha-2 a \alpha-\lambda}{1+i a} \\
& a_{0} \dot{(X)}=g(X) a_{1}(X)+h(X) a_{0}(X)-\frac{a_{1}(2 i \alpha-2 a \alpha-\lambda)}{1+i a}-2 a_{2}\left(\frac{1+i b}{1+i a} X^{3}-\frac{1-\alpha^{2}-i a \alpha^{2}-i \beta}{1+i a} X\right) \\
& g(X) a_{0}(X)=a_{1}(X)\left(\frac{1+i b}{1+i a} X^{3}-\frac{1-\alpha^{2}-i a \alpha^{2}-i \beta}{1+i a} X\right) ;
\end{aligned}
$$

Since $a_{i}(X)(i=0,1,2)$ are polynomials, $a_{2}(X)$ is constant and $h(X)=0$ are yielded from Eq.(17), we set $a_{2}=1$ as usual. Balancing the degrees of $g(X), a_{1}(X)$ and $a_{2}(X)$, we conclude that $\operatorname{deg}(g(X))=1$, therefore we have: $g(X)=A_{1} X+A_{0}$ is given. $a_{1}(X)$ is obtained from Eq.(18) as follow

$$
a_{1}(X)=\frac{1}{2} A_{1} X^{2}+A_{0} X-2 \frac{2 i \alpha-2 a \alpha-\lambda}{1+i a} X+R
$$

where $R$ is an integration constant.

$a_{0}(X)=\frac{A_{1}^{2} X^{4}}{8}+\frac{\left(3 A_{0} A_{1}-5 A_{1} \frac{2 i \alpha-2 a \alpha-\lambda}{1+i a}\right) X^{3}}{6}-R\left(\frac{2 i \alpha-2 a \alpha-\lambda}{1+i a}-A_{0}\right) X+R_{0}+\left(A_{0}^{2}+2\left(\frac{2 i \alpha-2 a \alpha-\lambda}{1+i a}\right)^{2}-3 A_{0}\left(\frac{2 i \alpha-2 a \alpha-\lambda}{1+i a}\right)+R A_{1}\right) \frac{X^{2}}{2}$

Substituting $a_{2}, a_{1}, a_{0}, g$, into Eq.(9) and setting all the coefficients of powers $X$ to be zero, then we obtain a system of nonlinear algebraic equations and by solving it, we obtain

$$
\begin{array}{ll}
A_{1}=\frac{1}{2} \sqrt{\frac{1+i b}{1+i a}} ; \quad A_{0}=\frac{8(2 i \alpha-2 a \alpha-\lambda)}{9(1+i a)} ; \quad R=R_{0}=0 \\
A_{1}=-\frac{1}{2} \sqrt{\frac{1+i b}{1+i a}} ; \quad A_{0}=\frac{8(2 i \alpha-2 a \alpha-\lambda)}{9(1+i a)} ; \quad R=R_{0}=0
\end{array}
$$

where $a \neq i, b$, and $\alpha$ are constants. Using the conditions Eq.(22)(23) and Eq.(9), we obtain the traveling wave solution of the Eq.(6)

$$
\begin{aligned}
\lambda=\frac{2-2 i \beta-2 \alpha^{2}-2 \alpha^{2} a^{2}}{a \alpha}, & M=\frac{1+i b}{1+i a} f(x, t)=-\frac{8 e^{\frac{i M^{1 / 4}(x+t)}{2}+2 \sqrt{2}}}{2 \sqrt{M} e^{4 \sqrt{2}+i M^{1 / 4}(x+t \lambda)}-1} ; \quad f(x, t)=-\frac{8 e^{\frac{i M^{1 / 4}(x+t)}{2}+2 \sqrt{2}}}{2 \sqrt{M} e^{4 \sqrt{2}}-e^{i M^{1 / 4}(x+t \lambda)}} \\
u(x, t) & =e^{\alpha x+\beta t} f(x, t) . \\
f(x, t) & =-\frac{2 \sqrt{2} i}{\sqrt{M}} \operatorname{coth}\left[\frac{-M^{1 / 4}(x+t \lambda)+4 \sqrt{2} i}{2}\right] \sqrt{\sqrt{M}-\sqrt{M} \tanh \left[\frac{-M^{1 / 4}(x+t \lambda)+4 \sqrt{2} i}{2}\right]^{2}} \\
f(x, t) & =\frac{2 \sqrt{2} i}{\sqrt{M}} \operatorname{coth}\left[\frac{M^{1 / 4}(x+t \lambda)+4 \sqrt{2} i}{2}\right] \sqrt{\sqrt{M}-\sqrt{M} \tanh \left[\frac{M^{1 / 4}(x+t \lambda)+4 \sqrt{2} i}{2}\right]^{2}} \\
u(x, t) & =e^{\alpha x+\beta t} f(x, t) .
\end{aligned}
$$

\section{Summary}

The evolution solutions for thecomplex Ginzburg-Landau equation are obtained by using the first integral method, which is based on the theory of commutative algebra. The simulation figures are 
shown with the corresponding parameters. The first integral method, which can be applied to nonintegrable equations as well as to integrable ones, is a standard, direct and computerizable method.

\section{References}

[1] T.Bohr, M. H. Jensen, G. Paladin, and A. Vulpiani, Dynamical Systems Approach to Turbulence (Cambridge University, New York), 1998.

[2] G. Dangelmayr, and L. Kramer, Mathematical Approaches to Pattern Formation in Evolution of Spontaneous Structures in Dissipative Continuous Systems, edited by F. H. Busse and S. C. Mu ller (Springer, New York), 1998.

[3] L. M. Pismen, Vortices in Nonlinear Fields (Oxford University/Clarendon Press, Oxford/New York), 1999.

[4] H. Haken, Synergetics (Springer, Berlin, Heidelberg 1983 ).

[5] C.O. Weiss, Spatio-temporal structures. Part II. Vortices and defects in lasers, Phys. Rep., 219, 311338 (1992).

[6] K. Staliunas, Laser Ginzburg-Landau equation and laser hydrodynamics. Phys. Rev. A, 48, 15731581 (1993).

[7] 8. P.K. Jakobsen, J.V. Moloney, A.C. Newell, R. Indik, Spacetime dynamics of wide-gain-section lasers, Phys. Rev. A, 45, 81298147 (1992).

[8] G.K. Harkness, W.J. Firth, J.B. Geddes, J.V. Moloney, E.M. Wright, Boundary effects in large-aspect-ratio lasers, Phys. Rev. A, 50, No 5, 43104322 (1994).

[9] W. Malfliet, W. Hereman, The tanh method. I: Exact solutions of nonlinear evolution and wave equations. Physica Scripta 1996;54:563.

[10] E. Yusufog, A. Bekir, Exact solutions of coupled nonlinear evolution equations. Chaos Soliton Fract 2008;37(3):842.

[11] AM. Wazwaz, The tanh method for travelling wave solutions of nonlinear equations. Appl Math Comput 2004;154(3):713.

[12] M. Inc, M. Ergut, Periodic wave solutions for the generalized shallow water wave equation by the improved Jacobi elliptic function method, Appl. Math. E-Notes 5 (2005) 89-96.

[13] T.C. Xia, B. Li, H.Q. Zhang, New explicit and exact solutions for the Nizhnik-Novikov-Vesselov equation, Appl. Math. ENotes 1 (2001) 139-142.

[14] A. Bekir, The tanh-coth method combined with the Riccati equation for solving nonlinear equation. Chaos Soliton Fract, 40 (2009) 1467-1474.

[15] Z. Sheng, The periodic wave solutions for the $(2+1)$-dimensional Konopelchenko-Dubrovsky equations, Chaos Solitons Fractals 30 (2006) 1213-1220.

[16] P. J. Olver, Applications of Lie group to differential equations (Springer,New York) 1993.

[17] T.R. Ding, C.Z. Li, Ordinary Differential Equations, Peking University Press, Peking, 1996.

[18] H. Jafaria, A. Soorakia, Y. Talebia, A. Biswas, The first integral method and traveling wave solutions to Davey-Stewartson equation, Nonlinear Analysis: Modelling and Control, 2012, Vol. 17, No. 2, 182-193.

[19] N. Taghizadeh, M. Mirzazadeh, F. Farahrooz, Exact solutions of the nonlinear Schrodinger equation by the first integral method, J. Math. Anal. Appl. 374 (2011) 549-553. 